\title{
Modelos sobre las Disoluciones Electrolíticas en la Formación Inicial de Profesores
}

\author{
Quira A. Sanabria, Royman Pérez y Rómulo Gallego \\ Universidad Pedagógica Nacional, Facultad de Ciencias y Tecnología, Departamento de Química, \\ calle 72 No. 11-86 Bogotá, D.C.-Colombia, \\ (e-mail: qsanabria@gmail.com, royman@pedagogica.edu.co, rgallego@pedagogica.edu.co)
}

\section{Resumen}

Este artículo muestra los fundamentos conceptuales y metodológicos y los resultados de un proyecto de investigación con profesores de química en formación inicial, realizado en el Departamento de Química de la Universidad Pedagógica Nacional, en Bogotá-Colombia. Se postula que en los primeros semestres de los programas de formación inicial de profesores en ciencias y en particular de Química se ha de propiciar un ambiente didáctico y pedagógico; trabajar sobre conceptos, modelos de las sustancias y su comportamiento. Se analiza en particular el caso de los modelos elaborados sobre disoluciones electrolíticas en medio acuoso. Se abordó el estudio como una investigación de carácter cualitativo. Se destaca de la metodología usada, los procesos de socialización tales como la concertación de trabajo individual y grupal.

Palabras clave: didáctica de las ciencias, disoluciones electrolíticas, formación de profesores, modelos en química

\section{Models of Electrolytical Dissolutions in Teacher's Initial Training}

\begin{abstract}
This article describes the conceptual and methodological basis, as well as the results of a research project with chemistry's teachers in their initial training at the Chemistry Department of the National Pedagogical University in Bogotá-Colombia. It is assumed that during the first semester of the initial formation program for teachers of basic sciences, and in particular of chemistry, a didactic and pedagogical environment must be created, and work must be done on substances and their behaviour. The elaboration of models about electrolyte dissolution in aqueous media was used as a study case and was developed as a qualitative research. One aspect of importance in the methodology used is the socialization processes, expressed in individual and group forms.
\end{abstract}

Keywords: didactics of science, electrolyte dissolutions, teachers training, chemistry models 


\section{INTRODUCCION}

Las relaciones históricas entre las ciencias de la naturaleza y los saberes tecnológicos hablan en favor de que los productos de las investigaciones científico tecnológicas afectan las relaciones entre las personas (Gieré, 1992); algo que se ha intensificado en el mundo actual. En consecuencia, los profesores de ciencias, en general, y de química, en particular, han de tener en cuenta estas relaciones, con miras a una aproximación de las nuevas generaciones a ese mundo. Desde esta perspectiva, en los últimos años, un número importante de investigadores en didáctica de las ciencias ha considerado relevante la caracterización de los procesos de enseñanzaaprendizaje de los profesores en formación que subyacen a los proyectos curriculares de los programas de licenciatura, por considerar que la dinámica que se desarrolla en los espacios académicos de formación, son comparables con los procesos que han caracterizado la producción de los paradigmas científicos (Mosquera, 2003). Tales construcciones se cimientan en la elaboración de modelos epistemológicos que se construyen a partir de la historia escrita de las ciencias naturales (Aduríz-Bravo e Izquierdo, 2002).

Los estudios elaborados desde finales del Siglo XX, pone de manifiesto la necesidad de trabajar no solo con estudiantes de diferentes niveles de enseñanza sino en particular con profesores en formación inicial en ciencias, en este caso, de química (Galagovsky et al., 2009; Gallego, 2004; Pessoa de Carvalho, 2004; Lombardi, 1998). Las concepciones en torno a la historia, la epistemología y la didáctica de las ciencias, han cambiado, lo que demanda una revisión de los proyectos curriculares sobre los que se direcciona la profesionalización de los profesores en formación inicial. Hoy por hoy se admite que las ciencias han sido producto de comunidades de especialistas, de conformidad con sus entornos culturales, políticos y económicos (Barona, 1994). Las diversas reconstrucciones históricas sobre hitos de las ciencias (Niaz, 2009) muestran que han sido, son y serán obra de seres humanos, no conformadas por verdades absolutas. Los hitos en química, como cualquier otra disciplina, se han caracterizado por un desarrollo que dista de ser lineal, aunque se admite que este desarrollo se evidencia en sus avances, independientemente del tiempo que pase para que se consolide. Aspecto que se puede establecer desde la versión de desarrollo científico de Kuhn (2000).

Los avances en química de manera particular, se reconocen por las diversas reestructuraciones, mas que sustituciones, de los sistemas de modelos que se han ido elaborando a través del tiempo (Kuhn, 2000). Admitidos como representaciones de una parte de la naturaleza, hecha objeto de estudio, no representan a la naturaleza en sí. Son construcciones abstractas producto de comunidades de especialistas que corresponden a la realidad que le ha sido asignada por la comunidad que estudia dichas entidades (Aduríz et al., 2005; Gieré, 1992), su finalidad fundamental es explicar el comportamiento de lo que no es perceptible por los sentidos, por ejemplo, la estructura y comportamiento de las sustancias; las diferentes explicaciones que se han elaborado al respecto a través de las épocas, muestran el uso de modelos. Éstos son susceptibles de ser categorizados evidenciando diversos niveles de complejidad como lo han publicado epistemólogos de las ciencias tales como: Caldín (2002), Del Re (2000), Tomasi (1999), entre otros.

Se afirma que los modelos en ciencias se convierten en el medio de comunicación por excelencia, los niveles de sofisticación de los mismos muestran desarrollos matemáticos que para el público no especializado significan más una dificultad que una posibilidad (Crawford, 2004). Es el caso de los modelos científicos para las disoluciones en general y las disoluciones electrolíticas en particular, cuyo examen de sus estructuras conceptuales y metodológicas, en un programa de formación inicial de profesores de química, contribuyen significativamente a un posicionamiento de tales características en lo tecnológico, que permite interpretar por ejemplo, que los electrolitos son base para la fabricación de productos como las fuentes portátiles de energía, que como mercancía, son adquiridas por las personas.

Las acotaciones presentes fueron las que fundamentaron la investigación adelantada con profesores de química en formación inicial, en el espacio académico Teorías químicas II. El proyecto fue adelantado dentro del programa de Maestría en Docencia de la Química, de la Universidad pedagógica Nacional, de Bogotá, DC. Colombia. 


\section{ACERCA DE LA CONSTRUCCIÓN HISTÓRICA Y EPISTEMOLÓGICA}

Al compararse los procesos de construcción de la historia de la Química a la luz de la historia interna y la historia externa (Lakatos, 1978) se puede establecer que la constitución de la química como una de las ciencias de la naturaleza, no siguió el mismo desarrollo conceptual y metodológico de la física (Bertomeu y García, 2006). De la misma manera que la categoría epistemológica de teoría (Lakatos, 1978) derivada de las elaboraciones newtonianas que no es aplicable en este caso. En la actualidad entender la historia de las ciencias como una serie de disciplinas es poco productivo intelectualmente Barona (1994), tal como sostener una discusión atemporal sobre autores, por tanto, cada vez es más importante la indagación de los contextos históricos y culturales en los que los científicos o comunidades de especialistas tomaron decisiones, aplicaron métodos de trabajo y seleccionaron temáticas objeto de estudio puesto que esta perspectiva permite interpretar los puntos de interconexión entre las concepciones científicas vigentes y las que se han abandonado, o que inician proceso de reflexión y análisis. Aspecto que muestra en sí una función didáctica, en la reconstrucción histórica de las ciencias, por hacer de la construcción colectiva del pensamiento científico su objeto de análisis.

En este proceso, los modelos científicos hacen parte de los objetos de análisis. Son representaciones altamente elaboradas de carácter abstracto que responden a un lenguaje particular, propio de la comunidad que los estructura. Su naturaleza es limitada, solo responden a una parte del fenómeno no a su totalidad. Construir explicaciones sobre un objeto de estudio demanda el uso de varios modelos que por si solos, no facilitan la fundamentación teórica (AdurízBravo et al., 2005). En los últimos años, algunos especialistas de la epistemología de las ciencias se han dedicado a elaborar categorizaciones de estas entidades, de acuerdo a las características que presentan, por ejemplo, Arca y Guidonni (1989) mencionan que todo individuo produce modelos. Por tanto estos pueden ser de sentido común, o modelos expertos según Aduríz-Bravo et al. (2005); estos modelos (no necesariamente científicos) pueden ser analógicos como los establecen Caldín (2002) y Tomasi (1999) que no representando el fenómeno, por comparación puede facilitar una interpretación del mismo. De hecho, los modelos que regularmente se usan en los procesos escolares muchas veces son reducciones de los modelos científicos, que pueden generar transposición didáctica, sin embargo, este aspecto no será desarrollado en este trabajo.

Considerando el cuerpo teórico de la química, se reconocen varios hitos en los que se enmarcan desarrollo de modelos particulares que favorecieron la consolidación de sistemas de modelos alrededor de conceptos estructurantes como la discontinuidad de la materia, el comportamiento eléctrico de la materia o los eventos termodinámicos. Caldin (2002), establece que los conceptos fundamentales en química se desarrollaron desde la mitad del siglo XVIII hasta nuestros días. En su análisis, ubica en primer lugar, lo concerniente a las sustancias. Menciona que los modelos construidos en este campo, comienzan en modelos de sentido común como los elaborados por Aristóteles, fundamentados en el trabajo empírico, y tendrán su siguiente modificación con las propuestas de Proust y Berthollet, cuando consideran que a la luz de la teoría de Dalton, hay sustancias que se combinan entre si en proporciones variables, en este caso, los modelos son más elaborados y por tanto, más abstractos. Con Lavoisier, se consolidan modelos de modelos, como el concepto de partícula y su intervención en la estructuración de las sustancias. El uso de modelos lingüísticos para la clasificación e interpretación de los elementos y los compuestos complejizan las explicaciones sobre el comportamiento de las sustancias. Aún en la actualidad, el trabajo publicado por Lavoisier es considerado como el origen de la química moderna. En segundo lugar, ubica las reacciones como fenómenos que establecen la composición y descomposición de sustancias, el tipo de modelo desarrollado es simbólico, con aspectos que han de involucrarse en las explicaciones, como los valores resultantes de la medición producto del uso de instrumentos. La balanza es uno de ellos; lo que permitirá posteriormente revalidar con argumentos los modelos estructurados sobre el flojisto (Bertomeu y García, 2006). La consolidación del cuerpo teórico sobre la estructura general de las sustancias se robustece con los trabajos de Dalton y sus modelos sobre átomos y moléculas.

Como se dijo anteriormente, intentar elaborar una explicación de cómo se han estructurado los sistemas de modelos de forma historiográfica no tiene sentido. Por tal motivo, si se aborda el sistema de modelos elaborados alrededor del comportamiento eléctrico de las sustancias, que se relaciona estrechamente con desarrollos tecnológicos para almacenar energía química en 
aparatos, que hoy se conocen con el nombre de acumuladores, vincula directamente con el sistema de modelos sobre las disoluciones electrolíticas. Aspecto importante de puntualizar desde su origen en el fenómeno disolución, asociado desde tiempos remotos con el acto de agregar agua (Tatton, 1973). Sin embargo, los modelos elaborados al respecto se distancian del fenómeno observable. Aspecto que los hace interesantes en particular cuando se busca construir una explicación para el fenómeno de la conducción de corriente por medio de disoluciones en las que intervienen sales y agua. Ya no es solo si la sal "desaparece" en el agua, es establecer lo que sucede a nivel submicroscópico con las entidades que constituyen las sustancias. Este constructo utiliza modelos asociados con la discontinuidad de la materia, se consolidó a la par con los modelos sobre las reacciones, requirió la introducción de un modelo distinto con respecto a las partículas como unidades estructurales de las sustancias -los iones- estableciendo otro tipo de conformación estructural más discreta. A este nivel, las explicaciones del fenómeno de conductividad eléctrica (Caldin, 2002), superan los modelos de orígen común, se amplió la visión frente a los aspectos que explican el comportamiento de las sustancias, ya no es solo cómo se conforman, sino, cómo se mueven, cómo se ordenan espacialmente, en qué proporción se combinan.

Grosso modo, los distintos modelos que han surgido para explicar el comportamiento de las disoluciones se centra en dos aspectos, el primero corresponde a la naturaleza eléctrica de las sustancias y su relación directa con la cantidad de sustancias, y el segundo tiene que ver con la explicación de reacciones que suceden en la formación de sales u otros compuestos. En esta dinámica se utiliza más de un modelo a la vez, que denotan el sentido macroscópico, microscópico y submicroscópico requerido según el nivel de interpretación del fenómeno seleccionado. El ejercicio de elaborar la reconstrucción histórica sobre los modelos que hacen parte de las explicaciones sobre el comportamiento eléctrico de la materia, permite sostener que en química no ha habido sustituciones radicales de sistemas de modelos o teorías para explicar el comportamiento de las sustancias (Khun, 2000), mas bien se han ido complejizando estas estructuras y la relación entre ellas.

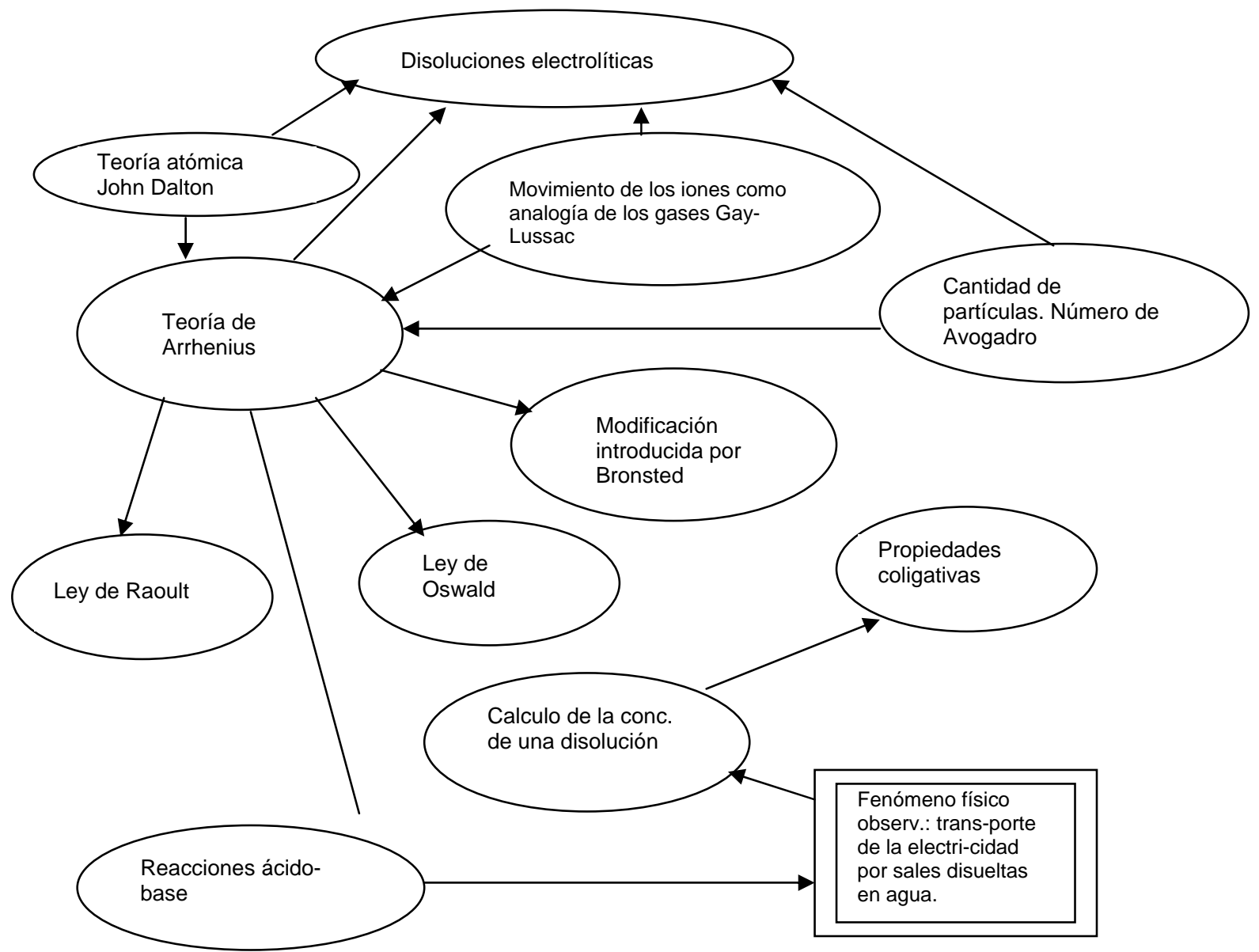

Fig. 1: Modelo de los Modelos del Comportamiento de las Disoluciones Electrolíticas (Sanabria, 2007) 
La figura 1 titulada Modelo de los Modelos del Comportamiento de las Disoluciones Electrolíticas, es el producto de la reconstrucción histórica hecha a partir de documentos de validez histórica y epistemológica para las explicaciones elaboradas sobre las disoluciones electrolíticas en medio acuoso. En este modelo se agrupan los distintos modelos que se han desarrollado y que en la actualidad tienen vigencia.

Es de destacar que esta red de modelos no es unidireccional, puesto que el conocimiento en ciencias no lo es, cada aporte teórico se convalida con la presencia del otro lo que muestra que dicha construcción mental no existe en la naturaleza y por lo tanto, requiere de un alto nivel de abstracción. Se trabaja con objetos teóricos producto del ingenio humano. La estructura de las sustancias y su comportamiento. Lo único que hace parte de la naturaleza es el fenómeno, que causa en primera instancia inquietud y es el vehículo para buscar la comprensión del mismo.

\section{SOBRE LA FORMACIÓN DE PROFESORES}

La necesidad de organizar la forma de enseñar para que mejore progresivamente la educación, es un propósito desde épocas pasadas. Mas la responsabilidad que eso se cumpla está depositada totalmente en el profesor (Pessoa de Carvalho, 2004) como un actor principal en el proceso, se sabe que no es suficiente con manejar algunos algoritmos, o tener simplemente la voluntad de hacer el servicio. Adicionalmente se necesita incluir estudios adelantados frente a los procesos de aprendizaje y en especial, los correspondientes a historia y epistemología de las ciencias (Gallego, 2007; Aduríz-Bravo e Izquierdo, 2002), que favorezca un examen de las concepciones histórico epistemológicas y didácticas, para elaborar modelos didácticos de los modelos científicos de la química (Galagovsky y Aduriz-Bravo, 2001). En lo tocante a los profesores de química en formación inicial, las investigaciones didácticas han puesto de presente errores en cuanto a las concepciones sobre las disoluciones en general, y de las electrolíticas en particular; errores como consecuencia de las experiencias escolares anteriores (Nappa et al., 2005; Jiménez et al., 2003). De Berg (2003), resalta que uno de los obstáculos epistemológicos evidenciados en estudios desarrollados sobre las concepciones muestra que muchos de esos errores provienen de las diferencias entre las explicaciones elaboradas a partir de las percepciones sensoriales, y las explicaciones elaboradas a partir de los modelos admitidos por los científicos. Aspectos que provocan en muchas ocasiones elaboraciones irreconciliables entre si.

En la formación de profesores es relevante el cambio de pensamiento frente al desarrollo histórico y epistemológico de las ciencias, por ser referencia del conocimiento como producto de la construcción colectiva en las distintas épocas, además del hacer de un profesor como mediador en la reconstrucción del constructo teórico con su sistema-aula (Quintanilla y Aduríz, 2006), a fin de que sus estudiantes se acerquen a este legado humano y lo transformen si ese es su interés y él haga retroalimentación de su ejercicio profesional y disciplinar. Por eso se afirma una vez más, que las ciencias basan su conocimiento en representaciones mentales que hacen de las ideas un medio para explicar el comportamiento de la naturaleza y transformarlo para beneficio de la humanidad (Caldin, 2002; Del Re, 2000; Tomasi, 1999; Gieré, 1992). Aunque en la literatura publicada sobre planes escolares se refleja la necesidad y pertinencia de las miradas retrospectivas de los proyectos curriculares a la luz de la historia y la epistemología contemporánea, son escasos los trabajos que consideren importantes los estudios de formación inicial de profesores usando en las metodologías de enseñanza el abordaje de las temáticas de trabajo desde los documentos publicados que tienen validez histórica, sobre la documentación de los textos seleccionados como guías. Dejando en un segundo plano el problema didáctico de la enseñanza superior de las ciencias.

De acuerdo con lo anterior, en los primeros semestres de los programas de formación inicial de profesores en ciencias y en particular de Química, de conformidad con los respectivos planes de estudio, se ha de propiciar un ambiente didáctico y pedagógico que haga posible la identificación, caracterización y discusión de esas concepciones o modelos elaborados sobre las sustancias y su comportamiento, con los cuales ingresan al proceso, para que se aproximen de manera admisible a los formulados y aceptados por la comunidad de especialistas. 
Metodológicamente el abordaje, que se realizó para esta investigación, con el grupo que se seleccionó, se centró en el trabajo colectivo como simulación de comunidades de especialistas quienes desarrollan sus propios sistemas de modelos sobre el objeto de estudio que les convoca. Posteriormente, se hizo una reconstrucción de la historia de dichos modelos, empleando los artículos originales en los que, la comunidad los propuso, los admitió, los trabajó, los modificó o los sustituyó por los aceptados en la actualidad. En esta reconstrucción se hizo indispensable intentar un análisis epistemológico que develara la concepción de ciencia y de química, subyacente a tales propuestas. En términos de la identificación y caracterización de las concepciones con las cuales ingresan al proceso estos profesores en formación inicial, como también las transformaciones paulatinas de ellas, se hizo un registro escrito y un seguimiento de las expresiones orales mediante las cuales manifiestan el lenguaje conceptual, metodológico y reelaboraciones de sus ideas sobre el comportamiento de las disoluciones electrolíticas y las implicaciones tecnológicas de las mismas.

Para ello se consideró como eje de trabajo los siguientes cuestionamientos: ¿Qué modelos han elaborado los profesores de química en formación inicial sobre disoluciones electrolíticas?; ¿Propicia una aproximación de los profesores de química en formación inicial a lo admitido por la comunidad de químicos, una estrategia didáctica centrada en lo histórico-epistemológico de modelos sobre disoluciones electrolíticas?; ¿Son los modelos estructurados por el profesor en formación punto de partida adecuado para la trasformación en términos de una aproximación a lo aceptado por la comunidad de químicos?

Para el análisis de lo evidenciado por los profesores en formación se establecieron unas categorías de análisis que se construyeron desde lo propuesto por epistemólogos de las ciencias; Según Del Re (2000), los modelos físicos, se aplican a objetos que son producto de la imaginación. Considerándose como producto del pensamiento científico, son descriptivos; los modelos matemáticos hacen parte de este grupo y son de carácter argumentativo. Advierte la importancia en la categorización en la medida que los modelos físicos cumplen diversa función dependiendo de cómo se aborde el mundo ordinario y las experiencias submicroscópicas de ese mundo, es decir, según se establezcan los valores de verdad. Al expresarlos lingüísticamente se necesita tener cuidado con los términos usados, para evitar definiciones innecesarias en razón a que los modelos físicos proveen una imagen del comportamiento de la naturaleza y se requiere determinar si el modelo es suficiente y la definición necesaria. En algunos casos la explicación es suficiente desde el punto de vista matemático mas no es familiar. De otro lado, Tomasi (1999) menciona que los modelos pueden ser clasificados en primera instancia, como material o abstracto que a su vez se categorizan como: Analógicos al corresponder a prototipos que si bien no son reales se parecen a estructuras o comportamientos de la realidad. Icónicos: Corresponden a modelos materiales que se han desarrollado en ingeniería, son gráficos. Y Simbólicos: corresponde a los modelos matemáticos. Los modelos materiales que se pueden clasificar en las categorías mencionadas anteriormente son aplicados en campos como la ingeniería y la arquitectura. Para el autor en referencia, los modelos analógicos se utilizan frecuentemente para explicar fenómenos sobre colisión atómica y molecular, reacciones bioquímicas, arreglo estructural de proteínas entre otros. La estructura básica de cualquier modelo químico es la teorización química, este modelo de modelos es susceptible de ser categorizado, sugiriendo cuatro grupos a tener en cuenta:

-Modelo Material: corresponde a esa porción material que se hace objeto de estudio que puede ser un fenómeno observable, el modelo puede ser una reducción o una simplificación del fenómeno que está limitado a las moléculas y a lo que suceda entre ellas (cantidad, reacomodación).

-Modelo Físico: se incluyen modelos que se relacionen con cantidad, es extensivo, se relaciona con fenómenos electrostáticos.

-Modelo Matemático: Incluye los aspectos relacionados con descripción e interacción física de un modelo material su principal desarrollo se relaciona con simulaciones computarizadas.

- Modelo Interpretativo: concreta aspectos establecidos en la aplicación matemática (modelo) del modelo material acorde con el modelo físico.

A su vez, establece niveles de complejidad que él denomina como simplicidad, consistencia en sí mismo, estabilidad, generalidad y utilidad. 
En el caso de Kretzenbacher (2003) y Caldin (2002), ellos mencionan los modelos materiales o físicos, lingüísticos e icónicos. Las tablas que se muestran a continuación (Tabla 1 y 2) establecen las categorizaciones con las que se clasificó los modelos que sobre disoluciones se hicieron objeto de trabajo en el aula .Se enfatizó en la construcción de modelos catalogados como interpretativos puesto que permiten la elaboración de explicaciones admisibles sobre las disoluciones electrolíticas. Conectan aspectos estudiados desde la aplicación matemática según el modelo material (Tomasi, 1999).

Tabla 1: Tipología para caracterización de los modelos elaborados por los profesores de química en formación inicial sobre disoluciones electrolíticas (Sanabria, 2007).

\begin{tabular}{|c|l|}
\hline MODELOS INTERPRETATIVOS & \multicolumn{1}{|c|}{ CARACTERISTICAS GENERALES } \\
\hline SIMBÓLICO & $\begin{array}{l}\text { Son modelos abstractos. Representado por entidades } \\
\text { matemáticas. }\end{array}$ \\
\hline MATERIAL & $\begin{array}{l}\text { Corresponde con la porción física del entorno a } \\
\text { estudiar, puede ser un fenómeno o una idea elaborada } \\
\text { del mundo. }\end{array}$ \\
\hline ANALÓGICO & $\begin{array}{l}\text { El énfasis está en su practicidad para explicar el } \\
\text { comportamiento de un sistema. Representado por } \\
\text { asociaciones con comportamientos o fenómenos del } \\
\text { entorno. }\end{array}$ \\
\hline MODELOS DESCRIPTIVOS & \multicolumn{1}{|c|}{ CARACTERISTICAS GENERALES } \\
\hline ICÓNICO & $\begin{array}{l}\text { Modelos materiales desarrollados, representados por } \\
\text { diagramas, dibujos, gráficos. Dependen del punto de } \\
\text { referencia. }\end{array}$ \\
\hline LINGÜISTICO & $\begin{array}{l}\text { Palabras que representan fenómenos o una porción de } \\
\text { la naturaleza. }\end{array}$ \\
\hline
\end{tabular}

Esta tipología de modelos a su vez, tiene cinco niveles de distinción que permiten establecer complejidad de los mismos, la tabla 2 muestra el criterio usado en cada caso.

Tabla 2: Niveles de distinción de los modelos según la tipología de modelos sobre disoluciones electrolíticas (Sanabria, 2007).

\begin{tabular}{|l|c|l|}
\hline No. & NIVEL DE DISTINCIÓN & \multicolumn{1}{c|}{ CARACTERÍSTICAS } \\
\hline 1 & SIMPLICIDAD & Sencillo y completo, reducido para elevar una característica relevante. \\
\hline 2 & $\begin{array}{c}\text { CONSISTENCIA EN SI } \\
\text { MISMO }\end{array}$ & $\begin{array}{l}\text { Su dimensión permite una explicación de la porción de la realidad acorde } \\
\text { con el cuerpo teórico. }\end{array}$ \\
\hline 3 & ESTABILIDAD & Permite cambios complementarios sin perder estructura teórica. \\
\hline 4 & GENERALIDAD & $\begin{array}{l}\text { Contiene conexiones con referentes diversos que no se consideraron } \\
\text { inicialmente. Comparten propiedades. }\end{array}$ \\
\hline 5 & UTILIDAD & $\begin{array}{l}\text { Hay congruencia con las entidades teóricas que le dan origen y aportan a } \\
\text { la explicación de fenómenos. }\end{array}$ \\
\hline
\end{tabular}

Cada modelo elaborado y caracterizado presentó varios niveles de distinción que evidenciaron trasformaciones que permitieron establecer modificaciones en cada instancia en la que se sometían a convalidación dentro del sistema-aula. Seguidamente se incorporan algunos registros seleccionados al azar de los modelos construidos por los profesores en formación con quienes se adelantó el trabajo, tanto al iniciar el proceso de intervención como al hacer seguimiento del mismo. Estos registros fueron el punto de partida para los análisis de las transformaciones conceptuales y metodológicas experimentadas por ellos, por el diseño y aplicación de la estrategia didáctica.

El modelo que se presenta fue elaborado en las instancias iniciales del proceso. El estudio de esta representación y de acuerdo con la bibliografía consultada, se clasificó como un modelo descriptivo y material que indica un componente icónico declarado, en cuanto al nivel de distinción es simple, consistente en sí mismo, general y útil puesto que se puede complejizar sin perder 
estructura. Puede funcionar como modelo analógico del comportamiento de las disoluciones muy a pesar de no reflejar la disociación de la sal, aquí lo significativo corresponde con la posibilidad de identificar las ideas alternativas o errores que sobre el tema ha elaborado en instancias escolares anteriores, que para el caso tiene que ver con el nivel de secundaria. El resto de los modelos elaborados no se distancian de lo observado en la figura 2.

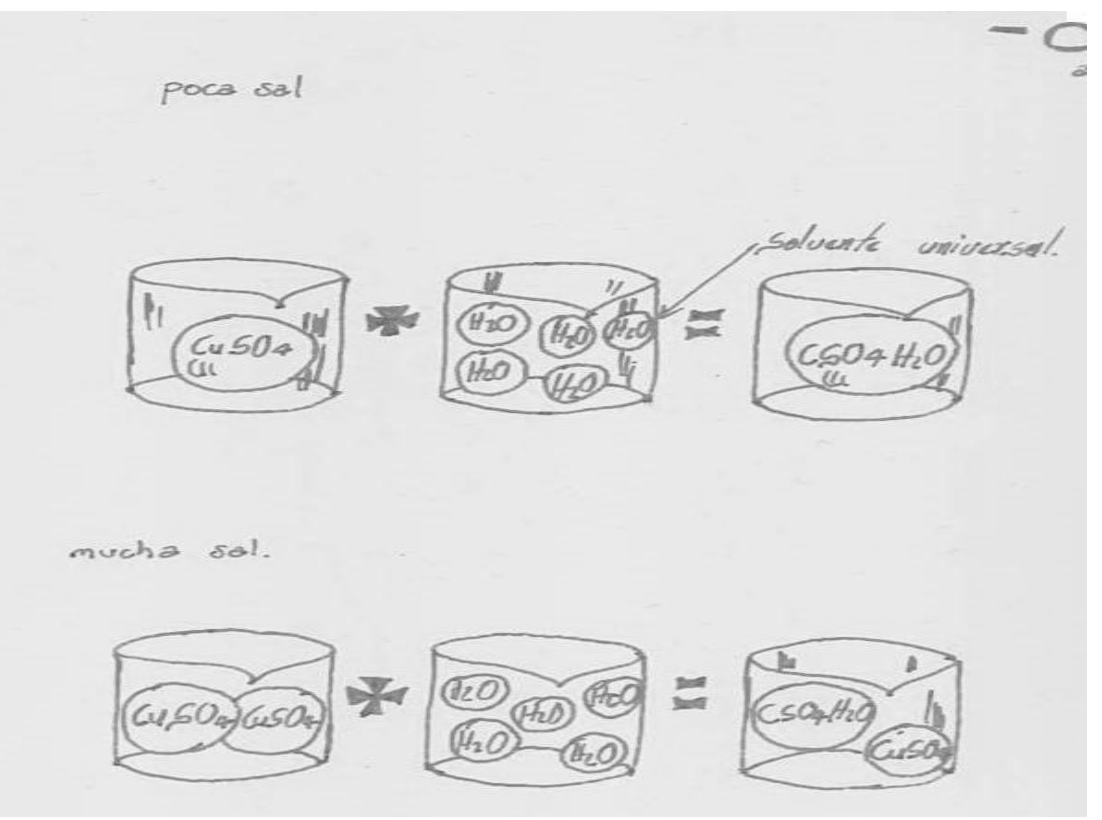

Fig. 2: Modelo Descriptivo sobre la Disolución de sulfato de cobre $\left(\mathrm{CuSO}_{4}\right)$ en agua.

Para el siguiente ejemplo, se trabajó con anterioridad documentos de validez histórica publicados por Raoult (1882), Arrhenius (1887), Ostwald (1888) y Brønsted (1923). Los ejemplos se tomaron tal como fueron elaborados, por considerarlos importantes como registro documental (figura 3 ).

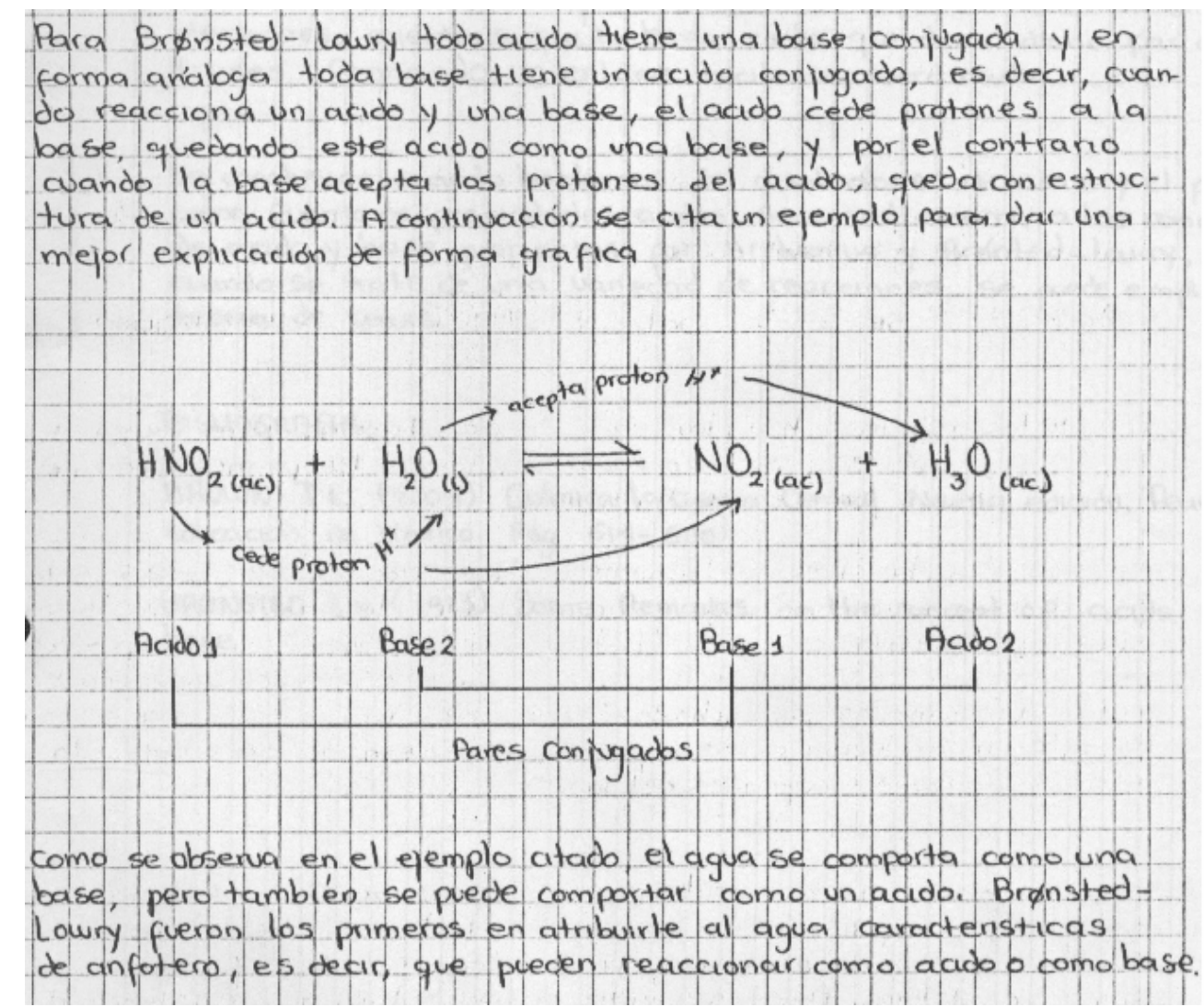

Fig. 3: Fragmento de composición escrita, sobre el desarrollo histórico del sistema ácido-base. 
Este fragmento de la composición elaborada sobre el desarrollo histórico del sistema de modelos ácido-base, muestra el uso de modelos lingüísticos y simbólicos que a diferencia del modelo de la figura 2, indican mayor nivel de complejidad al reconocer que son representaciones abstractas, aspecto que es producto del trabajo de lectura de documentos con validez histórica y epistemológica. Estos desarrollos se contrastaron mediante la elaboración de representaciones como los mapas conceptuales que muestran otros niveles de distinción no identificables en los casos anteriores. La figura 4 da cuenta de ello. El mapa que se analiza proviene de la instancia de trabajo individual previo a la socialización en comunidades o grupos.

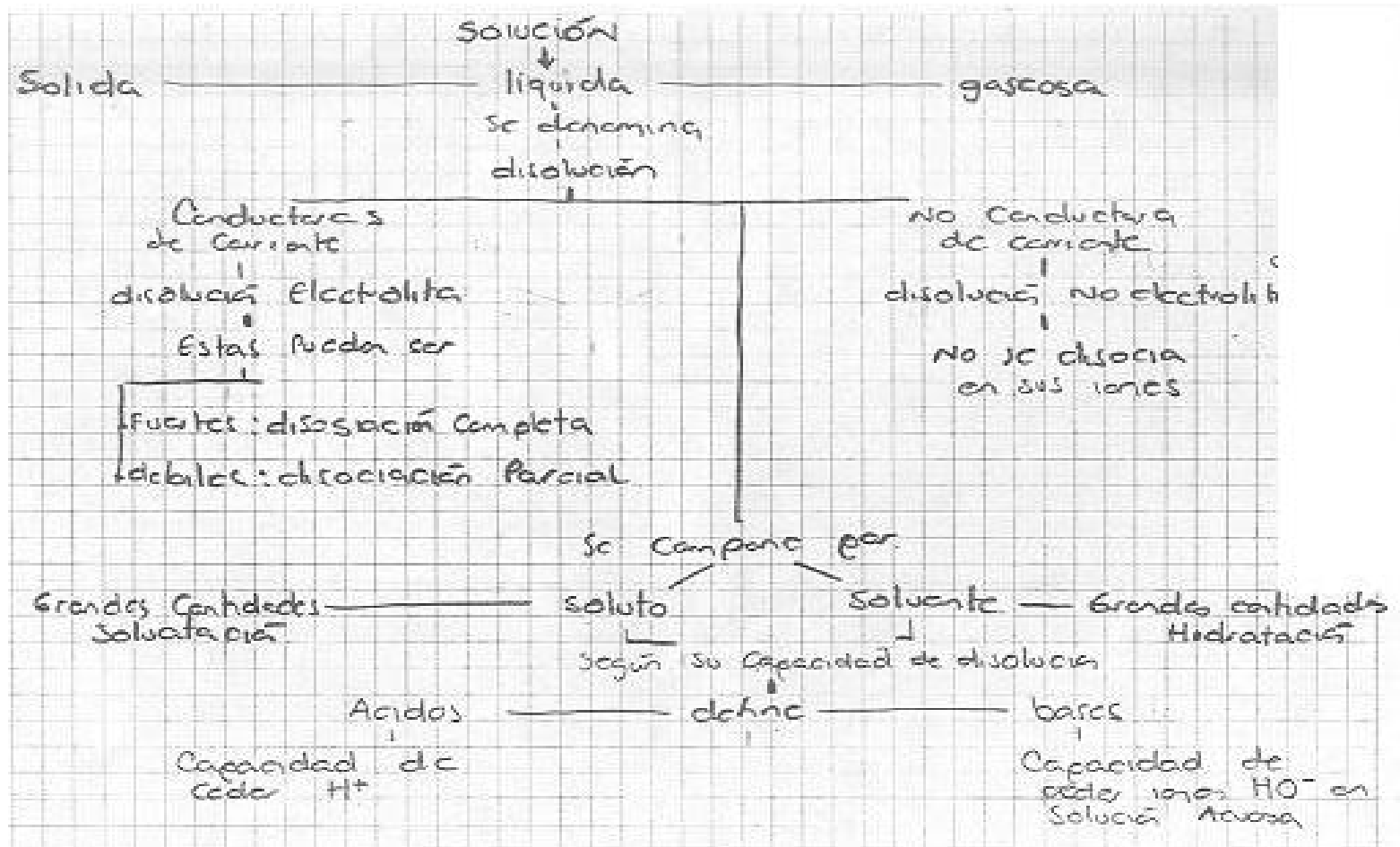

Fig. 4: Mapa conceptual elaborado sobre modelos lingüísticos de las disoluciones.

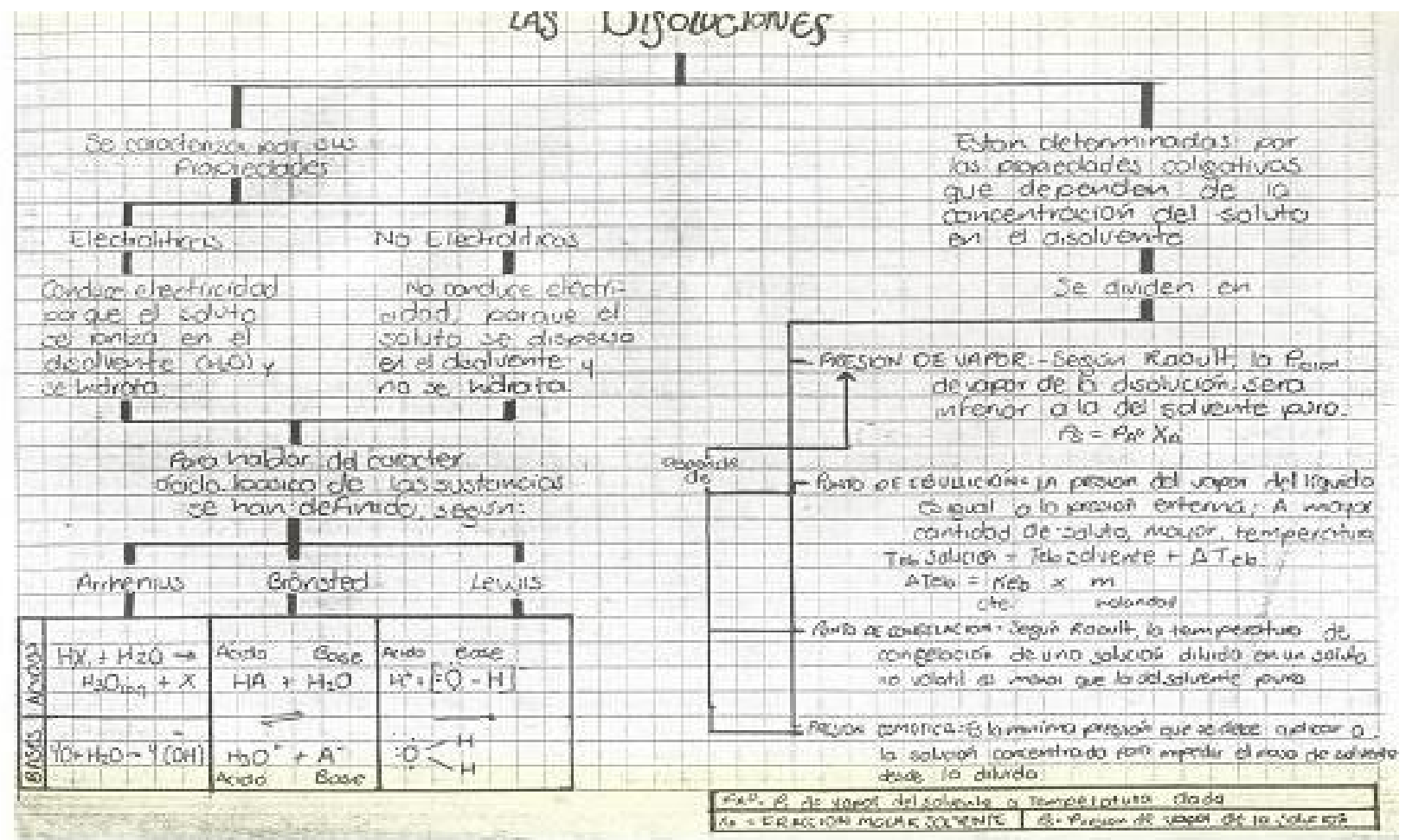

Fig. 5: Mapa conceptual elaborado sobre modelos lingüísticos de las disoluciones 
Un examen de este mapa elegido, muestra en su construcción fortalezas y debilidades al momento de considerar la relación de los modelos lingüísticos entre sí. Nótese la relación entre disolución (escrito solución) y ácido-base con el fenómeno de conducción de corriente. El hecho de cambiar los términos muestra una vez más, equivocaciones en los modelos elaborados. Sin embargo, permite establecer los niveles de jerarquización otorgados a cada término, que representa al modelo material que nos ocupa.

Posteriormente se le dio espacio a los grupos-comunidad para la reelaboración de sus mapas iniciales luego de las argumentaciones y discusiones colectivas. En general esos segundos mapas conceptuales presentaron un mayor nivel en sus estructuraciones. La figura 5 representa lo anotado anteriormente.

En este caso, se observa la inclusión de modelos simbólicos que permiten evidenciar una evolución del modelo de modelos de las disoluciones electrolíticas con respecto al mismo modelo material, los ácidos y las bases. Como es de conocimiento, una de las aplicaciones más importantes del cuerpo teórico de las disoluciones en general y las disoluciones electrolíticas en particular está en la interpretación de las reacciones en medio acuoso, el mapa conceptual que se analiza, destaca relaciones entre modelos que dan cuenta de ello.

\section{CONCLUSIONES}

Los resultados aquí expuestos, son sólo una muestra de la complejidad del sistema de modelos elaborados sobre disoluciones y la importancia de éstos para interpretar el comportamiento de las sustancias. Por ejemplo, los procesos de reacción y todo lo que podría decirse al respecto, acomodación espacial, transferencia electrónica, cambios termodinámicos, entre otros. Se tomaron únicamente aquellos modelos relacionados con el comportamiento de las disoluciones en medio acuoso, que permitieron reafirman las concepciones de los autores con respecto a los modelos científicos en Química, como representaciones de una parte de la naturaleza, susceptibles de convertirse en objeto de estudio, no obstante de ser construcciones abstractas producto de comunidades de especialistas sólo corresponden a un fragmento de la realidad.

Los modelos que pueden elaborar los profesores en formación inicial, aún a pesar de distanciarse de los modelos científicos vigentes, cumplen la función de representar el fenómeno estudiado, a medida que se socializan y se validan en los colectivos-aula, en vez de simplificarse, sucede lo contrario, se complejizan. Aspecto que permitió a la luz de las versiones epistémicas sobre las que se construyeron los criterios de análisis, caracterizarlos por naturaleza y niveles de complejidad. Evidenciando su función, en los procesos de comunicación, y de metacognición en un programa de formación inicial de profesores de química.

Del seguimiento descrito a las modificaciones y complejizaciones de los modelos que sobre disoluciones elaboraron los participantes de esta investigación, cabe resaltar la importancia que cobró en el proceso, el cambio actuacional y actitudinal frente al abordaje del desarrollo histórico y epistemológico de las ciencias, aspecto facilitador para interpretar el conocimiento científico como producto de una comunidad de especialistas. A su vez, la construcción de modelos hechos en las segundas instancias del proceso, a diferencia de las primeras, permite conjeturar que las trasformaciones conceptuales y metodológicas logradas con el grupo con el que se realizó la indagación pueden ser atribuibles a la estrategia didáctica diseñada e implementada.

Es posible elaborar modelos icónico- analógicos o simbólico- analógicos de los modelos científicos para las disoluciones en general y las disoluciones electrolíticas en particular, como un mecanismo concertador que facilite la comprensión consciente de los procesos que se hallan involucrados al abordar fenómenos de la naturaleza que tengan relación directa con estos modelos.

\section{REFERENCIAS}

Aduríz-Bravo, A. y M. Izquierdo: Acerca de la Didáctica de las Ciencias como disciplina autónoma. Revista Electrónica de Enseñanza de las Ciencias. ISSN 1579-1513 (en línea),1(3), (2002). http://www.saum.uvigo.es/reec/ Acceso: Agosto 25 (2005) 
Aduríz-Bravo, A., J. Garófalo, M. Greco e I. Galagovsky; Modelo Didáctico Analógico. Marco teórico y ejemplos. Número extra VII congreso Enseñanza de las Ciencias,1-6, (2005).

Arca, M. y P. Guidoni; Modelos Infantiles y Modelos Científicos Sobre La Morfología De Los Seres Vivos. Enseñanza de las Ciencias: 7(2), 162-167 (1989)

Arrhenius, S., On the dissociation of substances dissolved in water. Zeitschrift fur physikalische chemie 1(631),(1887). http://www.chemteam.info/Chem-History/Arrhenius-dissociation.html Acceso: septiembre 14 (2005)

Barona, J.; Ciencia e Historia: Debates y tendencias en la historiografía de la ciencia. Colección Scientia Veterum, $N^{\circ} 7$, Valencia, (1994)

Bertomeu S.J.R. y B.A. García; La revolución química. Entre la historia y la memoria, Universitat de València SC, Valencia (2006).

Brønsted, J. N. , Some Remarks on the Concept of Acids and Bases. Recueil des Travaux Chimiques des Pays-Bas, 42, 718-728 ,1923. http://chimie.scola.acparis.fr/Sitedechimie/hist chi/text origin /bronsted/Bronsted-Article.htm Acceso: Febrero 17(2009)

Caldin, E.; The structure of chemistry in relation to the philosophy of science. HYLE International journal for philosophy of chemistry, 8(2), 103-121, (2002). http://www.hyle.org/journal/issues/82/caldin.html Acceso: junio 10 (2006)

Crawford, B.A.; Supporting Prospective Teacher's conceptions of modeling in science. International Journal of Science Education: 26(11), 1379-1401 (2004).

Del Re, G.; Models and analogies in science. HYLE International journal for philosophy of chemistry. 6(1), 5-15, (2000). http://www.hyle.org/journal/issues/6/delre.htm Acceso: Junio 10 (2006)

De Berg, K.; The Developement of the theory of Electrolytic Dissociation. Science \& Education, 12, 397-419 (2003) (en línea) http://www.springerlink.com/content/×2010j4370×36649/ ) Acceso: Julio 30 (2009)

Gallego Badillo, R.; Un concepto Epistemológico de Modelo para la Didáctica de las Ciencias Experimentales. Revista Electrónica de Enseñanza de las Ciencias, ISSN 1579-1513 (en línea) 3(3), (2004). http://www.saum.uvigo.es/reec/ Acceso: Febrero 17(2009)

Gallego Torres, A.P.; Ciencia, historia, epistemología y didáctica de las ciencias: Las comunidades de especialistas. Revista Tecné, episteme y didaxis: 22, 113-125 (2007)

Galagovsky L. y A. Aduríz-Bravo; Modelos y Analogías en la enseñanza de las ciencias naturales. El concepto de modelo didáctico analógico. Enseñanza de las Ciencias: 19 (2), 231-242 (2001).

Galagovsky L., M. Di Giacomo y V. Castelo; Modelos vs. Dibujos: el caso de la enseñanza de las fuerzas intermoleculares, Revista electrónica de enseñanza de las ciencias, ISSN 1579-1513 (en línea) 8(1), (2009). http://www.saum.uvigo.es/reec/ Acceso : Febrero 28 (2009)

Gieré, R.N.; La Explicación de la Ciencia. Un Acercamiento Cognoscitivo. 1ª edición en español, Colección Ciencia Básica Consejo Nacional de Ciencia y Tecnología México (1992).

Jiménez, L.M., T.E. De Manuel y L.F. Salinas; El razonamiento Causal Secuencial en los Equilibrios Ácido -Base Múltiples: Propuestas Didácticas en el Ámbito Universitario. Enseñanza de las Ciencias: 21(2), 223-242 (2003). 
Kretzenbacher, H.L.; The aesthetics and heuristics of analogy. Model and metaphor in chemical communication. HYLE International journal for philosophy of chemistry: 9(2), 191-21 (2003). http://www.hyle.org/journal/issues/9-2/kretzenbacher.htm Acceso: Junio 10 (2006)

Kuhn, T.S.; La estructura de las Revoluciones Científicas. $5^{a}$ Reimpresión, Fondo de Cultura Económica, Bogotá, Colombia (2000).

Lakatos I.; La metodología de los programas de investigación científica. Alianza editorial, S.A. Madrid, España (1978).

Lombardi, O.; La Noción de Modelo en Ciencias. Revista Electrónica de Enseñanza de las Ciencias, ISSN 1579-1513 (en linea) 2(4). (1998). http://www.saum.uvigo.es/reecl Acceso: 16 Junio de 2006

Mosquera, C.; Consideraciones en torno a la formación de profesores de ciencias desde una perspectiva constructivista, Formación de profesores en América Latina: Diversos contextos sociopolíticos, Ediciones Antropos, pp 249-294, Bogotá D.C., Colombia (2003)

Nappa, N; M. Insausti y A. Sigüenza; Obstáculos para generar representaciones mentales adecuadas sobre la disolución, Revista Eureka sobre la enseñanza y divulgación de las ciencias,(en linea) 2(3), $2005.2 \quad$ http://www.apaceureka.org/revista/Volumen2/Numero 2 3/Nappa et al 2005.pdf Acceso: Febrero 17 (2009)

Niaz, M.; progressive transitions in chemistry teachers' understanding of nature of science based in historical controversias. Science \& Education (en línea) 18 43-65 (2009) http://www.springerlink.com/ content/963w615367027262/ Acceso: Julio 30 (2009)

Ostwald, W. F., Ostwald's dilution law. Zeitschrift für physikalische Chemie (en línea) 2, pp. 36-37. (1888) http://www.chemteam.info/Chem-History/Ostwald-1888.html Acceso: Julio 30 (2009)

Pessoa De Carvalho, A.; Formación de Profesores: Es Necesario que la Didáctica de las Ciencias Incluya la Práctica de la Enseñanza. Educación Química. 15(1) .pp. 15-22 (2004).

Quintanilla, M. y A. Aduríz; Enseñar ciencias en el nuevo milenio. Retos y propuestas, 11-13., Ediciones Universidad Católica de Chile, Santiago, Chile (2006)

Raoult, F. General Law of the Freezing of Solutions. [as translated and published by Henry M. Leicester \& Herbert S. Klickstein, A Source Book in Chemistry 1400-1900] (Cambridge, MA: Harvard, 1952),(1882) http://web.lemoyne.edu/ giunta/raoult.html Acceso: Julio 30 (2009)

Sanabria, Q.; Modelos sobre disoluciones electrolíticas. Implicaciones en la formación de profesores de química, Tesis de Maestría, Departamento de Química, Universidad Pedagógica Nacional, Bogotá D.C., Colombia (2007)

Tatton, R.; Historia General de las ciencias, Vol 2. Tomo III, 283-291. Ed.Destino, Barcelona, España (1973)

Tomasi, J.; Towards 'chemical congruence'of the models in theoretical chemistry, HILE International journal for the philosophy of chemistry, 5(2), 79-115 (1999). http://www.hyle.org/journal/issues /5/tomasi.htm Acceso : 19 febrero (2009) 